\title{
Ethische Sichtweise auf hochpreisige Arzneimittel
}

Eckhard Nagel, Dennis Henzler und Michael Lauerer

Inhaltsverzeichnis

\subsection{Einleitung -52}

4.2 Ethische Grundlagen im Gesundheitswesen - 54

4.2.1 Markt und Regulierung - 54

4.2.2 Gerechtigkeitsüberlegungen bei hochpreisigen Arzneimitteln - 55

4.3 Zu hohe Arzneimittelpreise? - 56

4.3.1 Fallbeispiel: Spinale Muskelatrophie (SMA) - 56

4.3.2 Fallbeispiel: Hepatitis C - 59

4.4 Fazit -59

$$
\text { Literatur - } 60
$$




\section{- Zusammenfassung}

Die Zulassung und Preisbildung neuer Arzneimittel ist in Deutschland, wie in vielen anderen Ländern, reglementiert. Damit fügen sie sich in die umfänglichen Vorgaben zur Strukturierung des Gesundheitswesens ein, die im Wesentlichen das Ziel haben, die Interessen des Leistungsanbieters, der Versichertengemeinschaft als Kostenträger sowie der behandlungsbedürftigen Personen adäquat zu berücksichtigen. Diese Ausgleichsfunktionen sind einer Reihe ethischer Normen unterworfen, die zum Teil explizit das Gesundheitswesen charakterisieren oder häufig auch implizit das genannte Ziel realisieren sollen. Der Artikel rekapituliert relevante ethische Grundlagen der Gesundheitsversorgung, welche einen engeren Bezug zur Preisfindung bei Medikamenten aufweisen. Dabei bedient er sich zweier Fallstudien anhand derer erkennbar wird, wie sich die Zusammenhänge zwischen Normensetzung und Verantwortung auf diesem Gebiet darstellen.

\subsection{Einleitung}

In Deutschland werden die Preise für Arzneimittel nicht den Marktmechanismen überlassen, sondern unterliegen der Beobachtung und - im internationalen Vergleich - eher strengen Regularien zur Vergütung und Preisbildung. Generell gehören die Gesundheitswirtschaft im Allgemeinen und der Arzneimittelmarkt im Speziellen zu den am stärksten regulierten Märkten in Deutschland (May und Bauer 2011). Die Verfahren zur Preisfindung für Arzneimittel können sicherlich nicht als perfekt gelten. Schon die juristischen Auseinandersetzungen in deren Folge deuten darauf (Stadelhoff 2016). Dennoch drängt sich - bis auf Ausnahmen - in der öffentlichen Wahrnehmung nicht der Eindruck auf, dass hohe Arzneimittelpreise eine wesentliche Herausforderung für die Gesundheitsversorgung sind. Das mag naheliegend auch an der vergleichsweise komfortablen Ressourcenausstat- tung des deutschen Gesundheitswesens liegen.

Sind im Hinblick auf den hohen regulatorischen und rechtlichen Aufwand, der im Arzneimittelmarkt betrieben wird, hierzulande also schon alle normativen Fragen adäquat adressiert? Die Antwort lautet nein. Vielmehr gibt es umfängliche Diskussionen über pharmazeutische Innovationen, deren Beurteilungsfähigkeit, die Schutznotwendigkeit für behandelte Personen, den Zugang und die Verteilung von Arzneimitteln und eben die Frage, wie im Hinblick auf den hohen Aufwand in der Entwicklung neuer Medikamente alle Forschungs- und Entwicklungskosten inklusive eines erwarteten Gewinns in den Preis eines Medikamentes adäquat einfließen können. Diese zuerst ökonomisch daherkommende Frage hat im Umfeld der Verteilung von Gesundheitsgütern in sich selbst aber einen normativen Grundtenor, sodass die Frage lautet: Ist der Preis von Arzneimitteln aus sich selbst heraus ethisch relevant? Hier lohnt sich der vergleichende Blick zum einen über die nationalen Grenzen hinaus, zum anderen auf spezifische Beispiele.

Beginnt man die Überlegungen mit dem Zugang zur Arzneimittelversorgung wird häufig vorgetragen, dass ein hoher bzw. zu hoher Arzneimittelpreis für sowohl die behandelten Menschen als auch für die Gesundheitssysteme negative Konsequenzen nach sich ziehen kann: Wenn z.B. durch hohe Arzneimittelpreise die Versorgungsqualität reduziert wird, liegt ein direkt negativer Effekt auf die Gesundheit von Individuen vor. Hier spielen die Vergütungssysteme eine zentrale Rolle: In vielen Ländern, in denen für Medikamente ein Selbstbehalt bezahlt werden muss, wirkt sich dies unmittelbar aus. Denn ein hoher Medikamentenpreis kann einen negativen Einfluss auf das Adhärenzverhalten bezüglich der Medikamenteneinnahme ausüben (Morgan und Lee 2017). Eine Erhöhung des Selbstbehalts, auch bedingt durch einen generellen Preisanstieg der Arzneimittel, kann zu einer Zunahme der Morbidität im Speziellen und zu schlechteren Gesundheits- 
outcomes im Allgemeinen führen (Kesselheim et al. 2015).

In Deutschland gibt es bei verschreibungspflichtigen Medikamenten einen differenzierten und eingeschränkten Selbstbehalt (BMG 2021): Volljährige zahlen pro Packung zehn Prozent des Preises, aber mindestens $5 €$ und höchstens $10 €$ sowie nie mehr als die tatsächlichen Kosten des Medikaments. Dabei sind die Zuzahlungen - unter Berücksichtigung des Eigenanteils für stationäre Behandlungen und bei Heilmitteln sowie häuslicher Krankenpflege - auf $2 \%$ des Bruttoeinkommens, für chronisch Kranke auf $1 \%$, beschränkt.

Ein hoher Arzneimittelpreis kann aber selbst in Ländern mit einer grundsätzlich vollständigen Kostenübernahme den Zugang $\mathrm{zu}$ einer bestmöglichen Gesundheitsversorgung infrage stellen: Dies ist der Fall, wenn bei einer flächendeckenden Einführung des hochpreisigen Medikaments die Bezahlbarkeit (affordability) durch die Versichertengemeinschaft nicht mehr gewährleistet ist. Ein aus der jüngeren Vergangenheit bekanntes Beispiel sind die antiviralen Therapien für Hepatitis C: Obwohl die Arzneimittel medizinisch effektiv und ökonomisch effizient sind, speziell bezogen auf individuelle Therapiekosten, galten die Auswirkungen auf das Arzneimittelbudget in mehreren Ländern - selbst in sogenannten High-Income-Countries - als belastend (Iyengar et al. 2016). Die Befürchtung wurde vorgetragen, dass die finanzielle Nachhaltigkeit des Gesundheitssystems durch zu hohe Arzneimittelpreise gefährdet werde.

Aber nicht nur bei häufig vorkommenden Erkrankungen, sondern auch bei der Pharmakotherapie von seltenen Krankheiten, die an Bedeutung gewinnt (Pomeranz et al. 2020), gibt es eine Diskussion über hochpreisige Arzneimittelbehandlungen: Für eine nachhaltige Finanzierung der Gesundheitssysteme ist auch die Ausgestaltung fairer und bezahlbarer Preise ein wichtiger Faktor (Merlini et al. 2020; Hagenbeek et al. 2020).

Letztendlich sind Fragen zu hohen Arzneimittelpreisen von ethischem Interesse, da sie in direkter Weise das moralisch bedeutsame Gut der Gesundheit betreffen. Normative Fragen zu Preisen drehen sich häufig um ein „,zu hoch“ oder ,zu niedrig“. ${ }^{1}$ In anderen Lebensbereichen stellen sich hingegen normative Fragen zu einem bestimmten Preis nicht: Niemand würde etwa ernsthaft über die Fairness hoher Restaurantpreise oder Smartphones diskutieren, da es sich - anders als bei Arzneimitteln - nicht um essentielle Güter handelt (Emanuel 2019). Gerade beim Zugang zu essentiellen Gütern besteht eine starke individuelle wie auch öffentliche Sensitivität. Hierin unterscheidet sich die Medizin von anderen Lebensbereichen.

Folglich ist die Toleranz in Bezug auf eine Ungleichheit bei der Versorgung mit Arzneimitteln gering. Was in anderen Bereichen an bestehender Ungleichheit akzeptiert wird, gilt im Bereich des Gesundheitswesens als inakzeptabel. Hintergrund ist die Konditionalität der Gesundheit, die in anderen Zusammenhängen auch als Transzendenz des Gutes Gesundheit bezeichnet wird (Kersting 2012): Das Gut Gesundheit ist gekennzeichnet durch einen „Ermöglichungscharakter“, ist also Voraussetzung für den „Genuss“ anderer Güter. Gesundheit hat nicht nur einen intrinsischen Wert (d.h. ist gut an sich), sondern auch einen instrumentellen Wert, welcher die Möglichkeiten determiniert, die einem Individuum im Leben offenstehen. Damit spielt sie eine entscheidende Rolle für die Erreichung von selbstbestimmten Lebenszielen (Hausman 2015). Da in dem Sinne Gesundheit primär nicht als natürliches Gut angesehen werden kann, sondern wesentlich durch gesellschaftliche Prozesse - wie dem Zugang zur Gesundheitsversorgung - geprägt wird, sind auch von der Gesellschaft eingesetzte Steuerungsmechanismen vonnöten.

1 Beispielhaft wäre hier der Preis der Arbeit, d. h. des gerechten Lohns, zu nennen. Ein zu niedriger Lohn wird als ungerecht angesehen. Aber auch zu hohe Gehälter werden als Gerechtigkeitsverletzungen wahrgenommen (vgl. Kiatpongsan und Norton 2014). 


\subsection{Ethische Grundlagen im Gesundheitswesen}

Die Gesundheitsversorgung im Allgemeinen und die Bedingungen der Arzneimitteltherapie im Speziellen sind unter ethischen Gesichtspunkten also mit Fragen der Gerechtigkeit verbunden. Dabei ist Gerechtigkeit zunächst ein vielschichtiger Begriff, der sich auf unterschiedliche Beziehungs- bzw. Tugendaspekte zwischen den Menschen bezieht (Aristoteles 2017). Generell zu differenzieren ist die Tauschgerechtigkeit, die sich auf reziproke sozioökonomische Beziehungen fokussiert. Die Verletzung gesellschaftlicher Normen und eine entsprechende Sanktionierung adressiert die korrektive Gerechtigkeit. Schließlich bemisst sich die distributive Gerechtigkeit an der Verteilung von Ressourcen und Lebenschancen. ${ }^{2}$

Die Hochpreisigkeit von Arzneimitteln tangiert zum einen Fragen der Tauschgerechtigkeit - zwischen dem Arzneimittelhersteller und den Kostenträgern bzw. der Versichertengemeinschaft. Zum anderen adressiert sie auch die Verteilungsgerechtigkeit - im Hinblick auf die Verfügbarkeit bzw. auf den Zugang zu Arzneimitteln.

Im Allgemeinen beziehen sich diese philosophisch-normativen Ansätze zur Gerechtigkeit auf das Sollen, d. h. auf die Rechtfertigung und Gültigkeit von Prinzipien. Im Gegensatz dazu existiert auch eine empirische Gerechtigkeitsforschung (siehe z. B. Dao Van et al. 2016). Diese geht etwa der Frage nach, welche Verteilungsregeln und -prinzipien von den Befragten bzw. Betroffenen als gerecht empfunden werden und wie diese Präferenzen begründet und motiviert sind. Ohne detaillierter auf den sogenannten naturalistischen Fehlschluss einzugehen, sei an dieser Stelle darauf hingewiesen, dass - ohne weitere Hinzufügung von normativen Prämissen - aus dem „Sein“ kein „Sollen“" abgeleitet werden kann. ${ }^{3}$

2 Für diese und folgende Ausführungen siehe auch Nagel (2020a).

3 Siehe zu diesem Gedanken allgemein Hume (1874).

\subsubsection{Markt und Regulierung}

Zu klären ist auch, welche Rolle Marktmechanismen im Hinblick auf die Gesundheitsversorgung spielen bzw. spielen sollen: Können Preise für Arzneimitteltherapien dem Zusammenspiel von Angebot und Nachfrage überlassen werden? Neben dem schon adressierten besonderen Stellenwert des Gutes Gesundheit ist bei der Beantwortung dieser Frage Gesundheit auch als ein unteilbares und öffentliches Gut zu verstehen: Dieses Gut umfasst alle Leistungen im medizinischen Bereich - von der Grundlagenforschung bis zur flächendeckenden Versorgung der Menschen - und wirkt sich auf andere Lebensbereiche aus. Für solche Güter ist der reine Markt ineffizient. Dies gilt gerade auch für die Abwehr sogenannter kollektiv-externer Gefahren (Kersting 2000). Dazu zählen etwa die Verschlechterung der gesundheitlichen Situation einer bestimmten Bevölkerungsgruppe oder auch Epidemien bzw. Pandemien - wie etwa aktuell die Ausbreitung des Coronavirus SARS-CoV-2. Ein weiterer Grund für das Versagen rein marktwirtschaftlich angelegter Strukturen im Gesundheitswesen ist die Informationsasymmetrie zwischen Kostenträgern, den Leistungserbringern und den Versicherten (Medvedeva 2007). In der Gesamtschau ist also ein Mindestmaß an staatlicher Regulierung unerlässlich (Lauerer et al. 2011).

Diese Einsicht führt auch im internationalen Vergleich zu verschiedensten Regulierungen, die die Finanzierung von Arzneimitteln nicht Marktmechanismen und somit den $\mathrm{Zu}-$ gang zu Arzneimitteln nicht der individuellen Zahlungsfähigkeit überlassen, sondern durch Bewertung und Verhandlung die verschiedenen - zum Teil konträren - Interessen auszugleichen versuchen: So dienen medizinische und ökonomische Analysen regelmäßig als Basis einer Preisfindung. Diese bewegt sich international im Kontinuum zwischen Verhandlungen über einen adäquaten Preis (z. B. primäre Strategie in Deutschland) bis hin zu Entscheidungen gegen die Finanzierung von als 
zu wenig kosteneffektiv erachteten Arzneimitteln (z. B. primäre Strategie in UK). Insbesondere sogenannten HTA-Institutionen, (Health Technology Assessment) obliegt es in vielen Ländern, die Grundlagen für eine solche Entscheidung $\mathrm{zu}$ schaffen und eine Bewertung ${ }^{4}$ der Kosten sowie des Nutzens eines Arzneimittels vorzunehmen.

Die Ergebnisse dieser Bewertungen alleine - z. B. in Form des Verhältnisses von Kosten und patientenrelevantem Nutzen - erlauben jedoch noch keine Aussage darüber, ob der vorgeschlagene Preis für ein Arzneimittel adäquat ist. Neben dem Verhältnis von Kosten und Nutzen sind vielmehr noch weitere (normative) Überlegungen in angemessener Art bei der Preisfindung zu berücksichtigen: So würde eine Entscheidung rein nach dem Kriterium der Kosteneffektivität etwa das Ziel der Maximierung des Outcomes bzw. des Nutzens bei begrenzten Ressourcen fokussieren. In einem solchen Fall werden aber entscheidende Facetten der Fairness außer Acht gelassen. Zum Beispiel besteht die Gefahr, den Schweregrad einer Erkrankung zu vernachlässigen. Auch kann es unter Umständen zur systematischen Benachteiligung bestimmter Gruppen - etwa Menschen mit einer Behinderung - kommen (Brock 2004). Daher gilt: Ein alleiniger Fokus auf das Kosten-Nutzen-Verhältnis eines Arzneimittels kann andere, ethische Prinzipien verdrängen, wie z.B. den Vorrang für die am meisten gefährdeten Menschen - also etwa Menschen mit einer besonders hohen Krankheitslast oder einem besonders hohen Risiko, an einer Erkrankung zu versterben (Emanuel 2019).

4 Genauer: Systematische, evidenzbasierte Bewertung medizinischer Verfahren bzw. Technologien bezüglich ihrer Effekte auf (patienten-)relevante Endpunkte in der Gesundheitsversorgung.

\subsubsection{Gerechtigkeitsüberlegungen bei hochpreisigen Arzneimitteln}

Bei der Findung eines fairen Preises für ein Arzneimittel müssen sowohl die legitimen Interessen des pharmazeutischen Unternehmens als auch die der „Käufer:innen“ berücksichtigt werden. Die primären Interessen des Herstellers betreffen die Amortisierung der Forschungs- und Entwicklungskosten eines Medikaments und die Erwirtschaftung eines Profits. Auf Nachfrageseite zählen der Wert des Medikamentes für das Individuum und die Finanzierbarkeit in ausreichender Menge sowie die Versorgungssicherheit auf kollektiver Ebene (z. B. Käufer:innen sind auf eine adäquate, zeitgerechte Lieferung angewiesen, was einen höheren Preis rechtfertigen kann), zu den zentralen Interessen (Moon et al. 2020).

Ein in der Fachliteratur ebenfalls kontrovers behandeltes Thema stellt die Ausgestaltung der Regulierung von Arzneimittelpreisen dar. Dabei fokussiert sich die Diskussion oft auf mögliche negative Auswirkungen der Regulation des Preises auf die Motivation zur Innovation, insbesondere der pharmazeutischen Industrie (Hughes 2020). In gleicher Weise wird eine gesetzliche Obergrenze von Arzneimittelpreisen mit der Begründung abgelehnt, dass eingeschränkte Gewinnerwartungen zu einer Reduktion industrieller Investition in die Forschung führen würden (Maitland 2002). Generell gibt es nachvollziehbarerweise einen engen Zusammenhang zwischen der Anzahl von einer Erkrankung betroffener Personen und der Umsatzwahrscheinlichkeit für ein Medikament. So finden sich im Bereich „HerzKreislauf-Erkrankungen“ regelmäßig vielfältige Entwicklungen, die einen großen Umsatz erwarten lassen. Genau umgekehrt ist es bei der Entwicklung von sogenannten „Orphan Drugs" für seltene Erkrankungen. Hier ist kein großer Mengenabsatz zu erwarten. Um dennoch Innovationen $\mathrm{zu}$ motivieren besteht eine gewisse Bereitschaft, hier hohe Preise zu akzeptieren, um Forschungen anzuregen und 
die Gesundheitsversorgung zu verbessern. Aus diesem Grund könnte eine undifferenzierte Regulierung das Forschungsinteresse mindern und auf lange Sicht zu einer relativ schlechteren Gesundheitsversorgung führen (Hughes 2020).

Vor diesem Hintergrund muss die Frage gestellt werden, auf welche Art und Weise faire Preise bestimmt werden können. Obwohl es zur Preisbildung bestimmte Standardtheorien gibt, finden diese auf dem Arzneimittelmarkt nur selten Anwendung (Hughes 2020). Grund dafür ist die starke Vulnerabilität der Nachfrager und die damit verbundene Unfähigkeit, einen Kauf des Arzneimittels abzulehnen: Transaktionsbeziehungen können als ausbeuterisch angesehen werden - wenn Akteur:innen ein Angebot aus rationalen Gründen nicht ablehnen können und andere Akteur:innen einen unverhältnismäßigen Nutzen aus diesem Umstand ziehen (Valdman 2009).

$\mathrm{Zu}$ hohe Preise für unentbehrliche und essentielle Güter, welche zur Befriedigung von Grundbedürfnissen ${ }^{5}$ dienen, werden als ungerecht angesehen - denn sie stellen letztlich eine Form der Ausbeutung von Hilfsbedürftigen dar. Emanuel (2019) vergleicht dies mit einer Situation, in der man von einer ertrinkenden Person vor deren Rettung zuallererst eine finanzielle Gegenleistung einfordern würde. In Erweiterung dieses Beispiels ist auf eine weitere Facette möglicher Ungerechtigkeit hinzuweisen: Da im Regelfall die Gesellschaft und nicht Einzelne für die teuren Arzneimittel aufkommen, wird das bestehende Solidaritätsgefühl innerhalb der Bevölkerung ausgenutzt und hohe Arzneimittelpreise werden damit begründet, dass sie das Leiden oder Sterben hinauszögern (Emanuel 2019).

\subsection{Zu hohe Arzneimittelpreise?}

Im Folgenden werden ausgewählte Fallbeispiele diskutiert, die die oben angeführten Überlegungen veranschaulichen. Das erste ausführliche Fallbeispiel fokussiert auf neue Gentherapie-Produkte zur Behandlung der Spinalen Muskelatrophie (SMA). Das ergänzende, zweite Fallbeispiel thematisiert weitere normative Facetten im Zusammenhang mit der Preissetzung von antiviralen Therapeutika für Hepatitis C.

\subsubsection{Fallbeispiel: Spinale Muskelatrophie (SMA)}

Für ein 2020 in Deutschland zugelassenes Gentherapie-Produkt zur Behandlung der Spinalen Muskelatrophie (SMA) ist einer der - bis dato - höchsten bekannten Arzneimittelpreise angesetzt worden: Die einzelne Spritze des Produktes, eines Gentherapeutikums, ${ }^{6}$ kostet hierzulande etwa zwei Millionen $€$. Indiziert ist das Medikament bei Kleinkindern mit der schwersten Form der SMA. Für diese Kinder geht der progressive Krankheitsverlauf damit einher, dass sie etwa nicht eigenständig sitzen können und sehr früh - in den ersten Lebensjahren - an der Erkrankung versterben. Erste Studienergebnisse zur Effektivität legen nahe, dass sich mit dem Gentherapeutikum eine Verbesserung der motorischen Fähigkeiten, Atmung und Schluckfähigkeiten erreichen lässt (Hofmann-Aßmus 2021).

Im Hinblick auf die genannten Kosten liegt die Frage nahe, wie sich der enorme Preis für ein einzelnes Medikament begründet. Ist es gerechtfertigt, in einem solidarisch finanzierten Versicherungssystem solche Summen für die Behandlung eines oder einer Einzelnen aufzuwenden oder ist es gar geboten? Bei der Suche nach Antworten dürften auch sogenannte Opportunitätskosten eine Rolle spielen: Mit dem

6 Hierbei handelt es sich um das Gentherapeutikum Zolgensma (Szent-Ivanyi 2021). 
gleichen Ressourceneinsatz wie für eine Spritze ist es möglich, etwa 40 Dialysepatient:innen für ein Jahr zu versorgen oder 250 schwere Hüftgelenksoperationen durchzuführen. Für andere hingegen wird sich die Frage nach der Finanzierung eines lebensrettenden Medikaments überhaupt nicht stellen: Wie kann der Preis zu hoch sein, wenn dadurch das Leben eines kranken Menschen deutlich verbessert, verlängert oder ein Kind sogar geheilt werden kann?

Beim konkreten Beispiel - der Einführung des Medikaments - steht aber nicht nur die (relative) Knappheit an finanziellen Ressourcen zur Diskussion. ${ }^{7}$ Vielmehr wurde von Seiten des Herstellers auch ein Engpass bei der Produktion des Medikaments (absolute Knappheit) kommuniziert. Im Hinblick darauf erklärte sich der Hersteller bereit, ein definiertes Kontingent des neuen Arzneimittels per Losverfahren an Erkrankte zu verteilen: Weltweit sollten 100 Kinder, die bestimmte Antragskriterien erfüllen, ausgewählt werden und das Medikament kostenlos noch vor der Zulassung erhalten. Dieses Vorgehen stellt eine in dieser Form noch nie dagewesene Art der Entscheidung über eine medizinische Therapie dar.

In diesem Konzept wird Knappheit auch dahingehend definiert, dass prioritär die Länder versorgt werden, in denen es bereits eine Zulassung für das Medikament gibt. Erst dann werden andere Länder weltweit identifiziert, in denen es die darüber hinaus zur Verfügung stehenden Medikamente zu verteilen gilt.

In diesem Beispiel stellt sich die Frage: Ist ein solcher Vorschlag ethisch akzeptabel?

Bei Fragen nach der Verteilungsgerechtigkeit ist es sinnvoll, gerade in der Medizin zuerst zu beantworten, inwieweit Knappheit tatsächlich absolut gegeben ist. Unter ethischen Gesichtspunkten ist dabei relevant: Gibt es z. B. unter der Maßgabe einer selektiven Kostenübernahme eine Priorisierung, die die angestrebte Gleichbehandlung unmöglich macht? Damit werden essentielle Fairness-Ar-

7 Folgende Ausführungen beziehen sich auf Nagel (2020b). gumente nicht berücksichtigt, denn es erfolgt eine Bevorzugung von Erkrankten, für die eine Kostenübernahme (absehbar) sichergestellt ist. Hierbei ist durchaus in der Bewertung relevant, ob es sich um eine offene (explizite) Bevorzugung oder um eine verdeckte (implizite) Benachteiligung handelt. Im vorliegenden Fall konnten sich jene von der Erkrankung Betroffene, denen der Zugang über ein Refinanzierungsangebot nicht offenstand, für das Härtefallprogramm ,bewerben“.

Sie sind also im Vergleich a priori benachteiligt. Die Frage stellt sich, welche Gründe dazu führen und wer diese $\mathrm{zu}$ verantworten hat? Relevant sind dabei Gesichtspunkte, die sich auf das Zulassungsverfahren beziehen, die die Organisation der Finanzierung der Arzneimittel betreffen und die Frage, inwieweit anderweitige regulatorische Rahmenbedingungen bestehen, die eine faire Distribution auch in den jetzt benachteiligten Ländern bereits ermöglichen. ${ }^{8}$ Liegt die Begründung in zeitlich unterschiedlichen Zulassungsbedingungen auf Seiten der Hersteller (Zeitpunkt der Antragstellung), verantworten diese die Ungleichheit und müssen eventuelle Nachteile für Betroffene selbst ausgleichen. Sind die Zulassungsbehörden für zeitliche Differenzen verantwortlich, kommt man voraussichtlich $\mathrm{zu}$ einem anderen Schluss, wobei hier ethische Abwägungen unter Umständen mit juristischen Fragestellungen (z. B. Haftungsfragen) konkurrieren.

Verteilungsfragen richten sich immer an die Gerechtigkeit: Hierzu geben die Organisationsstrukturen im Gesundheitswesen, die sich international stark unterscheiden, grundlegende Antworten. Dabei gibt es einen übergeordneten Konsens, der nahelegt: Gesundheitsversorgung soll so realisiert werden, dass Diskriminierung nach Geschlecht, Religion, Herkunft, ethnischer Zugehörigkeit oder sozialem Status weitestgehend ausgeschlossen ist (Nor-

8 Siehe hier bspw. das in der deutschen Gesetzgebung vorgesehene „Compassionate Use“ Programm. Dieses sieht vor, dass der Hersteller das Medikament frei zur Verfügung stellt (BfArM 2021). 
heim 2016). Dagegen finden sich unterschiedliche Auffassungen z. B. bei Fragen nach dem Alter als Differenzierungskriterium. Bereits an diesem Punkt erkennt man die Abhängigkeit der realen Distribution von Gesundheitsgütern von den jeweils zugrundeliegenden ethischmoralischen Auffassungen einer Gesellschaft. Hier gibt es explizit keinen globalen Konsens (Nagel und Lauerer 2016).

Mit Blick auf das vorgestellte Härtefallprogramm lassen sich folgende Schlussfolgerungen ableiten:

Zwar finden sich in der wissenschaftlichen Auseinandersetzung Theorien der Gerechtigkeit, die das Los als ein Verfahren in Knappheitssituationen und dahingehend diskutieren, dass die Zufallsauswahl zu einem adäquaten Verteilungsergebnis führen kann. Allerdings besteht im wissenschaftlichen Diskurs weitgehend Konsens darüber, dass das Los alleine keine gerechte Zuteilung knapper Ressourcen darstellt. In der Medizin etablierte Kriterien - wie z.B. Dringlichkeit, Schweregrad der Erkrankung, individueller Bedarf aufgrund fehlender Alternativen und ggf. auch die Erfolgsaussicht der Therapie - bleiben in dem hier beschriebenen Vorgehen unberücksichtigt. Solche Kriterien sollten - zum Teil objektiv bewertet und zum Teil auf die Patient:innenperspektive rekurrierend - Berücksichtigung finden. Dafür sind im konkreten Kontext spezifische und messbare Indikatoren festzulegen und untereinander zu gewichten.

Fragen zur adäquaten Preisbildung beschäftigen die Gesundheitsökonomik seit deren Etablierung. Im interdisziplinären, wissenschaftlichen Diskurs wurde dabei zunehmend deutlich, dass eine sinnvolle Allokation von Mitteln in einem so reglementierten Bereich der Daseinsvorsorge zuvorderst dem medizinischen Bedarf folgen muss. Dies bedeutet, dass sich eine primäre Fokussierung auf Gewinne, wie sie etwa im produzierenden Gewerbe oder in der klassischen Dienstleistungsbranche üblich ist, aus prinzipiellen Überlegungen heraus verbietet. Mit der zunehmenden Ökonomisierung in der Gesundheitswirtschaft sind Inves- tor:innen auf einen Bereich gestoßen, der mit finanziell attraktiven Entwicklungen, wie einer älter werdenden Gesellschaft, zunehmenden chronischen Erkrankungen und dem medizinisch-technischen Fortschritt, eine Anziehung ausübt.

Das dieser Entwicklung zugrundeliegende Streben diskreditiert mehr als die dahinterstehenden Unternehmen. Es stellt den gesamten Prozess einer sich verbessernden Lebenswelt durch den medizinischen Fortschritt infrage. Denn Fortschritt setzt voraus, dass möglichst alle Betroffenen an den Erkenntnissen und Entwicklungen teilhaben können. Anstatt wie im genannten Beispiel eine Lotterie, braucht es die breite öffentliche Debatte über eine faire Verteilung von Ressourcen in der Medizin.

Auch wenn die individuellen Therapiekosten als sehr hoch anzusetzen sind, muss eine Erstattung weder ökonomischen noch ethischen Grundsätzen widersprechen. Prinzipiell sind Menschen auch bereit, weniger effektiven aber hochpreisigen Interventionen einen hohen Stellenwert beizumessen, wenn die Intervention auf die Behandlung einer schwerwiegenden Krankheit abzielt (Nord et al. 1999). Die von der Schwere der Erkrankung abhängige Zahlungsbereitschaft der Bevölkerung ist in diesem Sinne ein Element der Ausgestaltung fairer Preise. Jedoch bedeutet eine gegebene gesellschaftliche Zahlungsbereitschaft im Umkehrschluss nicht, dass ein Preis auch gerecht(fertigt) ist. Die Zahlungsbereitschaft stellt in einem solidarfinanzierten Gesundheitssystem also eine notwendige, aber keine hinreichende Bedingung zur gerechten Preisfindung dar: Dies wird offensichtlich, wenn ein Solidaritätsgefühl mit Leidenden zur Erzielung von Gewinnen ausgenutzt wird. Ebenso wird dem Alter eine ethische Bedeutung zugemessen: Nach der sogenannten „Fair-Innings“" Argumentation wäre es in diesem Sinne geboten, knappe Ressourcen aus Fairnessgründen primär Jüngeren, die von einer fairen Lebensspanne noch am weitesten entfernt sind, zukommen zu lassen (Williams 1997). 


\subsubsection{Fallbeispiel: Hepatitis C}

Hochpreisigkeit von Arzneimitteln bemisst sich nicht allein an den Kosten für eine Einzeldosis. Vielmehr kann auch ein kosteneffektives Medikament in Verbindung mit hohen Fallzahlen zu Finanzierungslücken führen. Ein Beispiel stellt die Entwicklung von antiviralen Medikamenten für die Behandlung von Hepatitis $\mathrm{C}$ dar.

Weltweit sind ca. 71 Mio. Menschen an einer chronischen Hepatitis-C-Infektion erkrankt, wobei die Krankheit rund 400.000 Tode jährlich verursacht (WHO 2020). In den westlichen Ländern gehört eine Hepatitis-C-Infektion zu den Hauptursachen für die Entwicklung einer Leberzirrhose sowie eines hepatozellulären Karzinoms und ist eine der zentralen Indikationen für eine Lebertransplantation (Zignego und Craxì 2008). Die als äußerst wirksam beschriebenen Mittel zur Hepatitis-C-Behandlung besitzen bei einer Behandlungsdauer von nur 8 bis 12 Wochen eine Heilungsrate von über $95 \%$ und verursachen weniger Nebenwirkungen als ihre weniger wirksamen Vorgänger (WHO 2020). Allerdings hat der hohe Preis dieser Medikamente in vielen Ländern die nachhaltige Finanzierung der neuen Therapie infrage gestellt (Iyengar et al. 2016). In diesem Kontext konnte die therapeutische Option nicht allgemein zur Verfügung gestellt werden: So wurde z.B. in Brasilien und initial auch in der Schweiz der Zugang auf Patient:innen mit schweren Verlaufsformen beschränkt (WHO 2016). Zusätzlich war der Zugang in USamerikanischen Medicaid-Programmen an unterschiedliche Bedingungen geknüpft (Barua et al. 2015): u. a. an eine andauernde Alkoholund Drogenabstinenz oder an die Behandlung bzw. Beratung durch eine fachärztliche Person für Infektionskrankheiten oder Gastroenterologie.

Obwohl die Arzneimittel nach unseren Maßstäben als kosteneffektiv gelten, ist in vielen Ländern bei umfassender Einführung nicht von einer - zumindest kurzfristig - nach- haltigen Finanzierbarkeit auszugehen: Da die Behandlung einer Vielzahl an Erkrankten in kurzer Zeit angeboten werden müsste, würde es zu signifikanten Ausgabensteigerungen kommen (Craxi et al. 2016).

In der Gesamtschau kann davon ausgegangen werden, dass die hohen Preise den Zugang zur Versorgung erschweren und damit auch eine signifikante Barriere zur vollständigen Eliminierung des Virus darstellen (Craxi et al. 2016). Insbesondere in Pflichtversicherungssystemen mit einem hohen Selbstbehalt für Arzneimittel besteht die Gefahr, dass der universelle Zugang zu medizinischen Leistungen, vor diesem Hintergrund, nicht sichergestellt werden kann. Dies ist besonders bei Arzneimitteln gegen Infektionskrankheiten wie Hepatitis C - von Bedeutung, da sozioökonomisch vulnerable Gruppen oftmals nicht nur keinen vollständigen Versicherungsschutz genießen, sondern in der Regel auch ein höheres Infektionsrisiko haben (Vernaz et al. 2018).

\subsection{Fazit}

Auf die Frage, ob die Preisfestsetzung von Medikamenten normative Kriterien beinhaltet, lautet die Antwort, dass umfangreiche ethische Werturteile unabdingbar mit der Preissetzung verbunden sind. Inwieweit diese die einzelne Person, z. B. Patient:innen, betreffen oder aber systemisch auf die Struktur des Gesundheitswesens einwirken und welche nationalen oder internationalen Konsequenzen damit verbunden sind, ist von der jeweiligen Indikation, der Schwere der Erkrankung, dem Alter der betroffenen Patient:innengruppe, dem Grundverständnis von Gesundheit, der Organisation des Gesundheitswesens sowie weiteren Diskriminierungskriterien abhängig. Das bedeutet aber auch, dass die Preisfindung und die Preisfestsetzung für ein Medikament keine schlichte mathematische Prozedur darstellt, sondern umfänglichen Reflexionskriterien unterworfen sein muss. 


\section{Literatur}

Aristoteles (2017) Nikomachische Ethik (Hrsg Krapinger G). Reclam, Stuttgart

Barua S, Greenwald R, Grebeley J et al (2015) Restrictions for medicaid reimbursement of sofosbuvir for the treatment of hepatitis $\mathrm{C}$ virus infection in the United States. Ann Intern Med 163(11):215-223

BfArM (2021) Härtefallprogramme / Compassionate Use. https://www.bfarm.de/DE/Arzneimittel/ Arzneimittelzulassung/KlinischePruefung/ CompassionateUse/_node.html. Zugegriffen: 11. Mai 2021

BMG (2021) Zuzahlung und Erstattung von Arzneimitteln. https://www.bundesgesundheitsministerium.de/ zuzahlung-und-erstattung-arzneimittel.html. Zugegriffen: 4. Mai 2021

Brock D (2004) Ethical issues in the use of cost effectiveness analysis for the prioritization of health resources. In: Kushf G (Hrsg) Handbook of bioethics. Springer, Dordrecht, S 353-380

Craxi L, Sacchini D, Refolo P et al (2016) Prioritization of high-cost new drugs for HCV: making sustainability ethical. Eur Rev Med Pharmacol Sci 20(6):1044-1051

Daniels N (2008) Just health. Meeting health needs fairly. Cambridge University Press, Cambridge

Dao Van M, Lauerer M, Schätzlein V et al (2016) Organallokation im Spannungsfeld zwischen Erfolgsaussicht und Dringlichkeit: Ein Discrete Choice Experiment zur Erhebung von Präferenzen in der Bevölkerung. Gesundheitswes 78(7):454-459

Emanuel EJ (2019) When is the price of a drug unjust? The average lifetime earnings standard. Health Aff 38(4):604-612

Hagenbeek A, Gribben J, Jäger U et al (2020) Fair pricing of innovative medicines: an EHA position paper. HemaSphere 4(5):e488

Hausman DM (2015) Valuing health: well-being, freedom, and suffering. Oxford University Press, Oxford, New York

Hofmann-Aßmus M (2021) Erste Real-World-Daten zur Gentherapie bei SMA. Pädiatrie 33:60

Hughes RC (2020) Pricing medicine fairly. Philos Manag 19(4):369-385

Hume D (1874) A treatise on human nature. Longmans, London

Iyengar S, Tay-Teo K, Vogler S et al (2016) Prices, costs, and affordability of new medicines for hepatitis $\mathrm{C}$ in 30 countries: an economic analysis. PLoS Med 13(5):e1002032

Kersting W (2000) Gerechtigkeitsprobleme sozialstaatlicher Gesundheitsversorgung. In: Kersting W (Hrsg) Politische Philosophie des Sozialstaats. Velbrück, Weilerswist, S 467-507

Kersting W (2012) Wie gerecht ist der Markt? Ethische Perspektiven der sozialen Marktwirtschaft. Murmann, Hamburg
Kesselheim AS, Huybrechts KF, Choudhry NK et al (2015) Prescription drug insurance coverage and patient health outcomes: a systematic review. Am J Public Health 105(2):e17-e30

Kiatpongsan S, Norton MI (2014) How much (more) should CEOs make? A universal desire for more equal pay. Perspect Psychol Sci 9(6):587-593

Lauerer M, Emmert M, Schöffski O (2011) Die Qualität des deutschen Gesundheitswesens im internationalen Vergleich. HERZ, Norderstedt

Maitland I (2002) Priceless goods: how should life-saving drugs be priced? Bus Ethics Q 12(4):451-480

May U, Bauer C (2011) Regulierungsinstrumente in der GKV-Arzneimittelversorgung. Eine ordnungspolitische Analyse. Wissenschaftliche Verlagsgesellschaft, Stuttgart

Medvedeva S (2007) Health-Technology-Assessment Ein Instrument zur Nivellierung von Informationsasymmetrien im Gesundheitswesen? In: v. d. Schulenburg J-MG (Hrsg) Das Gesundheitssystem zwischen Wettbewerb und Staatsdirigismus. Cuvillier, Göttingen, S 49-77

Merlini G, Gribben J, Macintyre E et al (2020) Access to affordable orphan medicines in Europe: an EHA position paper. HemaSphere 4(5):e477

Moon S, Mariat S, Kamae I et al (2020) Defining the concept of fair pricing for medicines. BMJ 368:14726

Morgan S, Lee A (2017) Cost-related non-adherence to prescribed medicines among older adults: a crosssectional analysis of a survey in 11 developed countries. BMJ Open 7(1):e14287

Nagel E (2020a) Gesundheit und Gerechtigkeit. In: von der Decken K, Günzel A (Hrsg) Staat - Religion Recht, 1. Aufl. Nomos, Baden-Baden, S 47-62

Nagel E (2020b) Lebensrettende Medikamente im Lostopf. Frankfurter Allgemeine, 14.03.2020. https:// www.faz.net/aktuell/wissen/medizin-ernaehrung/ verlosung-der-gentherapie-zolgensma-beispiellosertabubruch-16671698.html

Nagel E, Lauerer M (Hrsg) (2016) Prioritization in medicine. An international dialogue, 1. Aufl. Springer, Cham

Nord E, Pinto JL, Richardson J et al (1999) Incorporating societal concerns for fairness in numerical valuations of health programmes. Health Econ 8(1):25-39

Norheim O (2016) Ethical priority setting for universal health coverage: challenges in deciding upon fair distribution of health services. BMC Med 14(1):1-4

Pomeranz K, Siriwardana K, Davies F (2020) Orphan drug report 2020. https://www.evaluate.com/media/ 2741/download. Zugegriffen: 2. Juni 2021

Stadelhoff S (2016) Rechtsprobleme des AMNOGVerfahrens. Nutzenbewertung - Erstattungsbetrag Folgen. Nomos, Baden-Baden

Szent-Ivanyi T (2021) Zwei Millionen Euro für eine Infusion. Frankfurter Rundschau, 21.02.2021. https:// www.fr.de/politik/zwei-millionen-euro-fuer-eineinfusion-90213053.html. Zugegriffen: 17. März 2021 
Valdman M (2009) A theory of wrongful exploitation. Philos Impr 9(6): 1-14

Vernaz N, Calmy A, Hurst S et al (2018) A buyers' club to improve access to hepatitis $\mathrm{C}$ treatment for vulnerable populations. Swiss Med Wkly 148:w14649

WHO (2016) Global report on access to hepatitis C treatment. Focus on overcoming barriers. https://apps. who.int/iris/bitstream/handle/10665/250625/WHOHIV-2016.20-eng.pdf. Zugegriffen: 5. Juni 2021
WHO (2020) Hepatitis C - fact sheet. https://www.who. int/en/news-room/fact-sheets/detail/hepatitis-c. Zugegriffen: 19. Apr. 2021

Williams A (1997) Intergenerational equity: an exploration of the 'fair innings' argument. Health Econ 6(2):117-132

Zignego AL, Craxì A (2008) Extrahepatic manifestations of hepatitis $\mathrm{C}$ virus infection. Clin Liver Dis 12(3):611-636

Open Access Dieses Kapitel wird unter der Creative Commons Namensnennung 4.0 International Lizenz (http:// creativecommons.org/licenses/by/4.0/deed.de) veröffentlicht, welche die Nutzung, Vervielfältigung, Bearbeitung, Verbreitung und Wiedergabe in jeglichem Medium und Format erlaubt, sofern Sie den/die ursprünglichen Autor(en) und die Quelle ordnungsgemäß nennen, einen Link zur Creative Commons Lizenz beifügen und angeben, ob Änderungen vorgenommen wurden.

Die in diesem Kapitel enthaltenen Bilder und sonstiges Drittmaterial unterliegen ebenfalls der genannten Creative Commons Lizenz, sofern sich aus der Abbildungslegende nichts anderes ergibt. Sofern das betreffende Material nicht unter der genannten Creative Commons Lizenz steht und die betreffende Handlung nicht nach gesetzlichen Vorschriften erlaubt ist, ist für die oben aufgeführten Weiterverwendungen des Materials die Einwilligung des jeweiligen Rechteinhabers einzuholen.

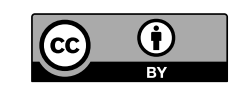

